\title{
Design of optical nor logic gates using two dimension photonic crystals
}

\author{
Wen-Piao Lin ${ }^{1, ~ *, ~ Y u-F a n g ~ H s u ², ~ H a n-L u n g ~ K u o ~}{ }^{1}$ \\ ${ }^{1}$ Electronic Engineering Department, Chang Gung University, Taoyuan, Taiwan \\ ${ }^{2}$ Electronic Engineering Department, Chienkuo Technology University, Changhua, Taiwan
}

\section{Email address:}

wplin@mail.cgu.edu.tw (W. Lin), hugogo7646@gmail.com (H. Kuo)

\section{To cite this article:}

Wen-Piao Lin, Yu-Fang Hsu, Han-Lung Kuo. Design of Optical Nor Logic Gates Using Two Dimension Photonic Crystals. Journal of Modern Physics. Vol. 2, No. 3, 2013; pp: 144-147. doi: 10.11648/j.ajmp.20130203.18

\begin{abstract}
In this paper, a novel all-optical NOR logic gate based on two dimension (2-D) photonic crystals (PC) is designed and simulated by a cascade of two all-optical switches. The new all-optical switch is composed of a nonlinear photonic crystal ring resonator (PCRR) and T-type waveguide. The PC structure has a square lattice of silicon rod with refractive index of 3.39 in air. The bandgap of this structure is derived by the plane wave expansion (PWE) method, which is from $\mathrm{a} / \lambda=0.32$ to $\mathrm{a} / \lambda=0.44$. In this structure to work at the wavelength of $1550 \mathrm{~nm}$, the lattice constant ' $\mathrm{a}$ ' should be $630 \mathrm{~nm}$; the total size of the proposed optical NOR gate is only $18 \mu \mathrm{m} \times 11 \mu \mathrm{m}$. The simulation results using two dimensional finite difference time domain (FDTD) method indicated that the proposed optical NOR logic gate is a potential candidature for ultrafast optical digital circuits.
\end{abstract}

Keywords: All-Optical NOR Logic Gate, Photonic Crystals (PC), Photonic Crystal Ring Resonator (PCRR), Plane Wave Expansion (PWE), Finite Difference Time Domain (FDTD)

\section{Introduction}

The problems of future computation and communication are unavoidable since conventional electronic technology will very soon reach its speed limit. The all-optical signal processing for the networks can handle large bandwidth signals and large information with very high speed. Ultrafast optical logic gates based on nonlinear photonic crystal (NPC) are the key device in the optical signal processing systems and future optical networks ${ }^{[1-4]}$. The two dimensional (2-D) NPCs have refractive index changes in two perpendicular directions that play an important role on the photonic components than one dimensional and three dimensional NPCs ${ }^{[5-7]}$. It is possible due to case in controlling the propagation modes, accurate calculation of photonic bandgap (PBG), efficient light confinement, simple design and easy fabrication capability. Beside, optical waveguide using photonic crystals have been the attractive attention because of their small dimension and low loss structure.

In recent years, all-optical logic gates ${ }^{[8-12]}$ have been reported many configurations such as a Mach-Zehnder interferometer based on self-collimation, periodically poled lithium noibate (PPLN) waveguide, photonic crystal self-collimated beam with a phase lag, semiconductor saturable absorber etalons, nonlinear ring resonators inserted between three parallel line defects, etc. These schemes have some fundamental limitations like latency time, power consumption, speed and size.

In this paper, a new all-optical NOR gate is proposed by a cascade of two all-optical switches. The all-optical switch using 2-D NPC is composed of a nonlinear photonic crystal ring resonator (PCRR) and T-type waveguide. Simulation has been performed through 2-D finite difference time domain (FDTD) method. This method is used to simulate electromagnetic wave propagation in any kind of materials in the time domain. Next, the PBG of the material can be calculated by plane wave expansion (PWE) method. The paper is organized as follows: In Sec. 2, we describe the structure design of proposed all-optical NOR logic gate in detail. Simulation results are discussed in Sec. 3. Finally, we conclude the article in Sec. 4.

\section{Structure Design}

In this paper the $15 \times 15$ square lattice $2-\mathrm{D}$ PC is used for designing the structure. The lattice constant, denoted by ' $a$ ', is $630 \mathrm{~nm}$ which is a distance between the two neighbor 
rods. The radius of rod is $0.21 \mathrm{a}$, which is close to $0.133 \mu \mathrm{m}$. The relative permittivity of the dielectric rods in the structure is $\varepsilon_{r}=11.5$ that is the refractive index of 3.39 . The circular Si rod is surrounded by air.

First, the plane wave expansion (PWE) method is used to calculate the photonic bandgap (PBG) of the PC structure. Fig. 1 shows the simulation of the band diagram of the structure without any defects, which gives the transverse electric (TE) photonic bandgap and propagation modes in the first Brillouin zone. The normalized frequency $(a / \lambda)$ is observed from 0.32 to 0.44 whose corresponding wavelength ranges from $1432 \mathrm{~nm}$ to $1968 \mathrm{~nm}$. The photonic crystal ring resonator (PCRR) is fabricated by the reduced expansion of photonic crystal, which embedded between two waveguides. The diameter of PCRR is $6 \mathrm{a}$. The waveguides in $\Gamma$-X direction are single mode in the whole PC bandgap shown in Fig. 2. In the corners of the PCRR there are four extra rods shifted toward corner of $0.707 \mathrm{a}$ with the same refractive index and radius. The rods are needed to omit the back scattering at the corner ${ }^{[13,14]}$. In order to confirm the switching property shown in Fig. 3, the structure is excited from port A with a Gaussian light source at the normalized resonant frequency of $0.406 \mathrm{c} / \mathrm{a}$, where $\mathrm{c}$ is the speed of light in vacuum. In this case, there is no signal at port $\mathrm{B}$ and all the energy is transmitted to port D.

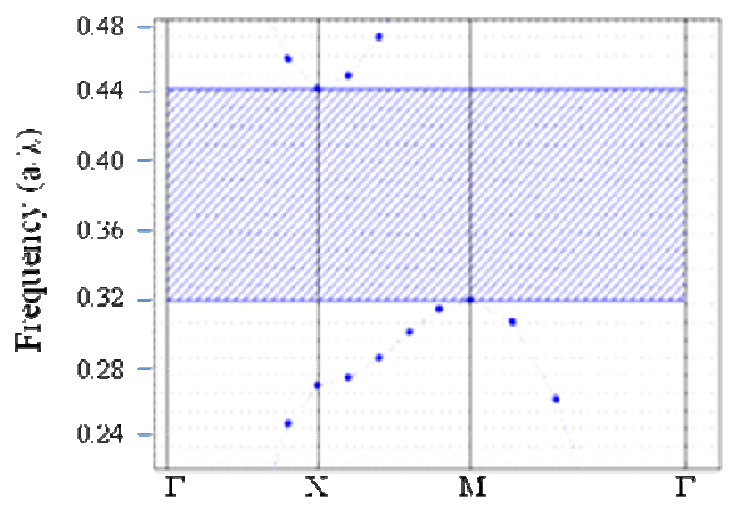

Figure 1. TE band diagram of $15 \times 15$ square lattice without introducing any defects.

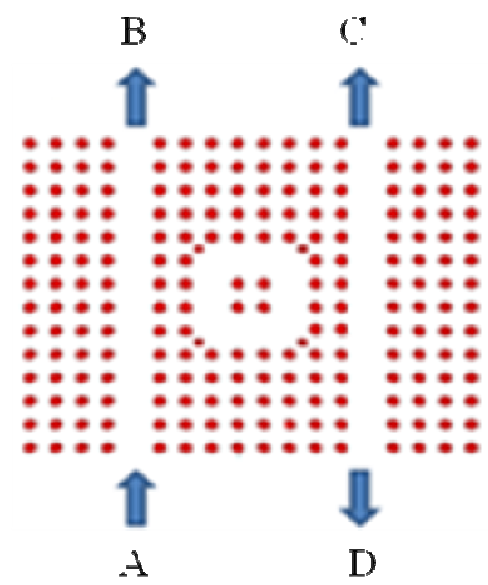

Figure 2. The schematic structure of photonic crystal ring resonator (PCRR).
Moreover, we propose a new structure of all-optical T-type switch consisted of T-type waveguide and PCRR shown in Fig. 4. The structure is excited from port $\mathrm{A}$ and exit from port $\mathrm{B}$, which control by port $\mathrm{C}$. When an optical signal is applied to port $\mathrm{C}$, no output signal is in port $\mathrm{B}$ due to the coupling of PCRR. On the contrary, the optical signal is transmitted to port B when no signal is applied to port C. The normalized transmission spectra of port B is obtained by conducting fast Fourier transform (FFT) of the fields that are calculated by multiple scattering method (MSM) shown in Fig. 5. From Fig. 5 we can see that this T-type optical switch has two switching resonant frequencies of $0.385 \mathrm{c} / \mathrm{a}$ and $0.425 \mathrm{c} / \mathrm{a}$. Moreover, the optical switch has a lower switching time of about 3 ps after simulation.

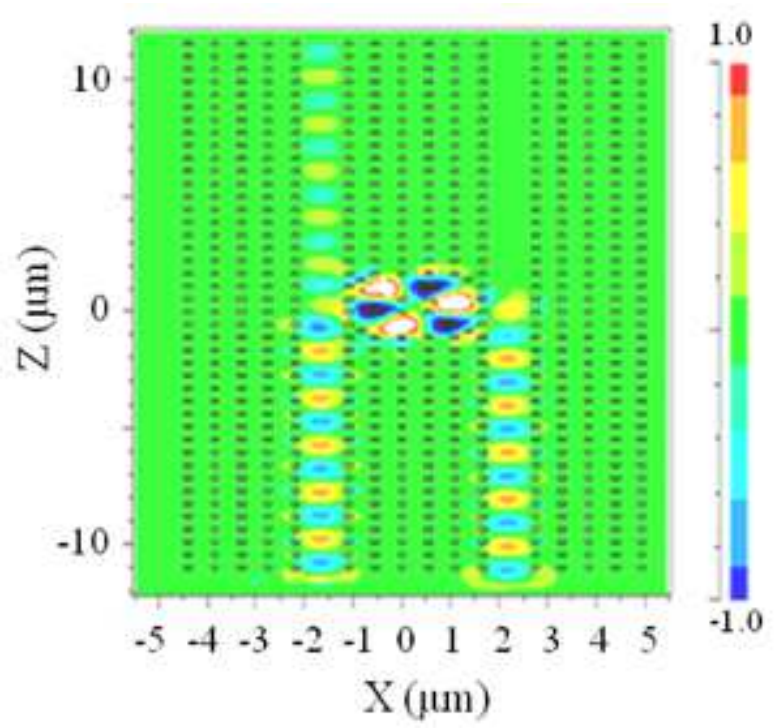

Figure 3. The electric field distribution in PCRR.

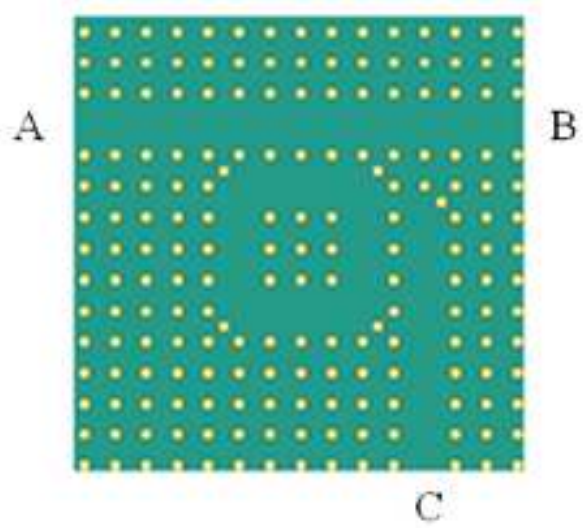

Figure 4. The structure of proposed all-optical T-type switch.

Finally, a novel all-optical NOR logic gate is designed by a cascade of two all-optical switches shown in Fig. 6. The center distance of both PCRR is $14 \mathrm{a}$. The total size of the present gate is about $18 \mu \mathrm{m} \times 11 \mu \mathrm{m}$, which is smaller than the conversational PC-based optical logic gates. This optical logic gate is excited from port $\mathrm{A}$ and exit from port B (i.e. the output of the logic gate), which control by port C and port $\mathrm{D}$ (i.e. the inputs of the logic gate). 


\section{Simulation Results and Discussions}

To conduct the Gaussian light source into the logic gate, the logic function can be achieved by the two optical switches operation. Moreover, the optical field distribution of the proposed all-optical NOR logic gate is obtained by conducting fast Fourier transform (FFT) of the fields that are calculated by FDTD method shown in Fig. 7. In Fig.

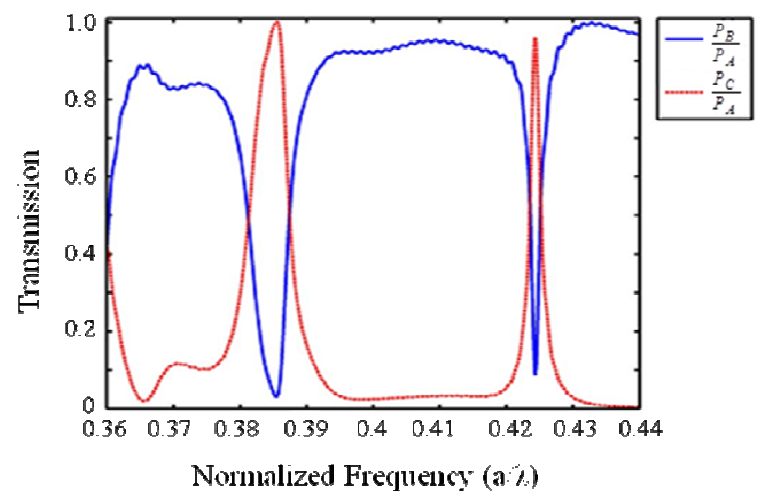

Figure 5. Transmission spectrum of all-optical T-type switch.

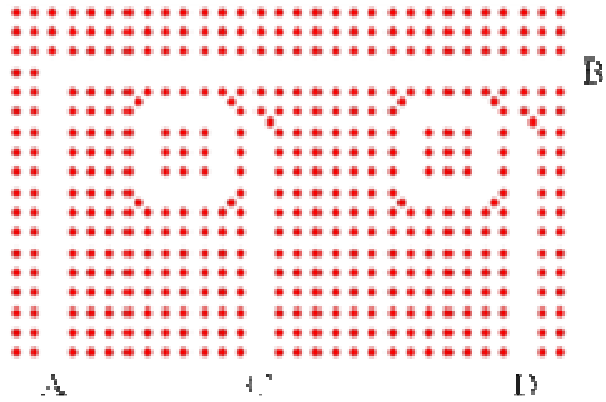

Figure 6. The structure of an optical NOR logic gate.

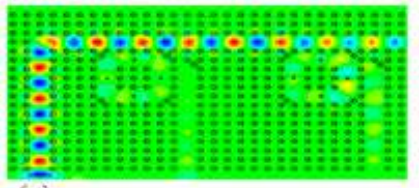

(a)
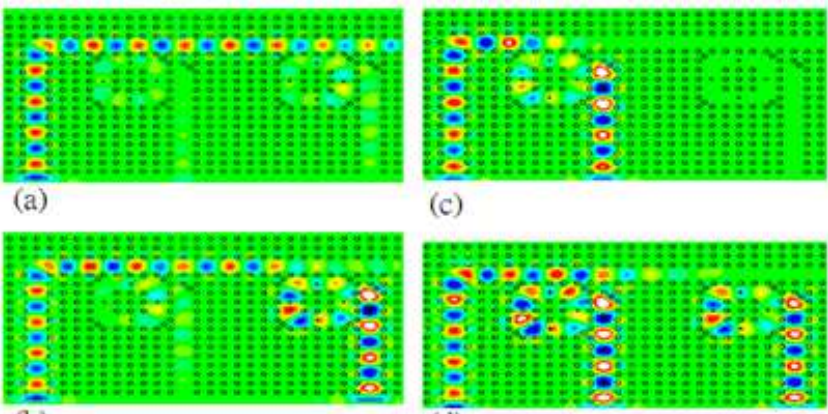

(b)

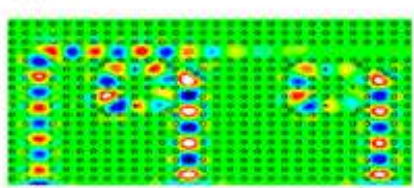

(d)

Figure 7. The electric field distribution of all-optical NOR logic gate at (a) no input at port $C$ and port $D$; (b) no input at port $C$ and applied input at port $D ;(c)$ applied input at port $C$ and no input at port $D$, and (d) applied input at port $C$ and port $D$.

7(a), when port $\mathrm{C}$ and port $\mathrm{D}$ be 'OFF', and port $\mathrm{A}$ is applied the pumping optical source, we can see that the optical signal can reach the port B. In Fig. 7(b), there is no optical signal output at port $\mathrm{B}$ due to second PCRR operation and turn off the optical channel under port $\mathrm{C}$ of 'OFF', port D of 'ON', and port A applied the pumping optical source. Moreover, port B is also no output under the changing status of port $\mathrm{C}$ and port $\mathrm{D}$ shown in Fig. 7(c). In

Fig. 7(d), when port $\mathrm{C}$ and port $\mathrm{D}$ be 'ON', the pumping optical source of port A is turn off at first PCRR operation and only $2 \%$ optical signal stay on the waveguide channel from port D. Finally, the summary of operation results for the proposed optical NOR logic gate shows in Table 1.

\section{Conclustion}

We have proposed an novel all-optical NOR logic gate based on 2-D PC. This gate is consisted with two optical T-type switches. The T-type switch adopts a T-type wave guide in $\Gamma$-X direction with a nonlinear PCRR, which can be achieved a fast switching time of about 3 ns. Moreover, the structure of proposed NOR gate has small size of about 18 $\mu \mathrm{m} \times 11 \mu \mathrm{m}$ and can work with low power consumption in comparison with the conventional PC-based optical gate. Therefore, the proposed optical NOR logic gate is very useful to apply on ultrafast optical logic operations and future optical computing components.

Table 1. All-optical NOR logic gate truth table

\begin{tabular}{ccc}
\hline Input of Port C & Input of Port D & Output of Port B \\
\hline 0 & 0 & 1 \\
0 & 1 & 0 \\
1 & 0 & 0 \\
1 & 1 & 0 \\
\hline
\end{tabular}

\section{Acknowledgements}

This work was supported by the National Science Council of R.O.C. under Contract NSC 99-2221-E-182-021-MY3 and the High Speed Intelligent Communication (HSIC) Research Center of Chang Gung University, Taoyuan, Taiwan.

\section{References}

[1] E.-H. Lee, "Micro/nano-scale optical network: A new challenge toward next generation", Proc. Int. Conf. on Transparent Opt. Networks, 4 (2008), 118-119.

[2] S. Robinson and R. Nakkeeran, "A bandpass filter based on 2D circular photonic crystal ring resonator", Proc. IEEE Int. Conf. on Wireless Opt. Commun. Networks 1 (2010), 1-3.

[3] Riadh Bchir, Afraph Bardaoui, Hatem Ezzaouia,"Design of silicon-based two-dimensional photonic integrated circuits: XOR gate", IET Optoelectron (2013), Vol. 7, Iss. 1, pp. $25-29$

[4] Yi-Pin Yang, I-Chen Yang, Chia Hsien Chang, Yao-Tsung Tsai, Kun-Yi Lee, Yi-Rung Tsai, Yong-Si Tu, Sin-Fu Liao, Ching-Chou Huang, Yen-Juei Lin, Wei-Yu Lee, Cheng-Che Lee,"Binary Operating in All-Optical Logic Gates Based on Photonic Crystals",2012 Internatioinal Symposium on Computer,Consumer and Control 
[5] A. P. Kabilan, X. S. Christina and P. E. Caroline, "Design of optical logic gates using photonic crystal", Proc. Int. Conf. on Internet (2009), 1-4.

[6] T.-T. Shih, Y.-D. Wu and J.-J. Lee, "Proposal for compact optical triplexer filter using 2-D photonic crystals", IEEE Photon. Technol. Lett. 21 (2009), 18-20.

[7] Y. D. Wu, K. W. Hsu, T. T. Shih and J. J. Lee, "New design of four-channel add-drop filters based on double resonant cavity photonic crystals", J. Opt. Soc. Am. B 26 (2009), 640-644.

[8] D. Zhao, J Zhang, P. Yao, X. Jiang and X. Chen, "Photonic crystal Mach-Zehnder interferometer based on self-collimation", Appl. Phys. Lett. 90 (2007), 231114-231114-3.

[9] J. Wang, J. Sun and Q. Sun, "PPLN based flexible optical logic AND gate", IEEE Photon. Tech. Lett. 20 (2008), 211-213.
[10] Y.-L. Zhang, Y. hang and B. Li, "Optical switches and logic gates based on self-collimated beams in two-dimensional photonic crystal”, Opt. Express 15 (2007), 9287-9292.

[11] C. Porzi, M. Guina, “A. Bogoni and L. Poti, All-optical nand/nor logic gates based on semiconductor saturable absorber etalons", IEEE J. Sel. Top. Quantum Electron 14 (2008), 927-937.

[12] P. Andalib and N. Granpayeh, "All-optical ultracompact photonic crystal AND gate based on nonlinear ring resonators", J Opt. Soc. Am B 26(2009), 10-16.

[13] M. Djavid, A. Ghaffari and M. S. Abrishamian, "Coupled-mode analysis of photonic crystal add-drop filters based onring resonators", J. Opt. Soc. Am. B 25 (2008), 1829-1832.

[14] V. D. Kumar, T. Srinivas and A. Selvaraian, "Investigation of ring resonators in photonic crystal circuits", Photonics and Nanostructures 2 (2004), 199-20 